\title{
Anisotropic Feature Extraction from Endoluminal Images for Detection of Intestinal Contractions
}

\author{
Panagiota Spyridonos $^{1}$, Fernando Vilariño ${ }^{1}$, Jordi Vitrià ${ }^{1}$, \\ Fernando Azpiroz ${ }^{2}$, and Petia Radeva ${ }^{1}$ \\ ${ }^{1}$ Computer Vision Center and Computer Science Dept. \\ Universitat Autonoma de Barcelona, Barcelona, Spain \\ ${ }^{2}$ Hospital Universitari Vall d'Hebron, Barcelona, Spain
}

\begin{abstract}
Wireless endoscopy is a very recent and at the same time unique technique allowing to visualize and study the occurrence of contractions and to analyze the intestine motility. Feature extraction is essential for getting efficient patterns to detect contractions in wireless video endoscopy of small intestine. We propose a novel method based on anisotropic image filtering and efficient statistical classification of contraction features. In particular, we apply the image gradient tensor for mining informative skeletons from the original image and a sequence of descriptors for capturing the characteristic pattern of contractions. Features extracted from the endoluminal images were evaluated in terms of their discriminatory ability in correct classifying images as either belonging to contractions or not. Classification was performed by means of a support vector machine classifier with a radial basis function kernel. Our classification rates gave sensitivity of the order of $90.84 \%$ and specificity of the order of $94.43 \%$ respectively. These preliminary results highlight the high efficiency of the selected descriptors and support the feasibility of the proposed method in assisting the automatic detection and analysis of contractions.
\end{abstract}

\section{Introduction}

Small intestine (SI) contractions are among the motility patterns which bear very high clinical pathological significance for many gastrointestinal disorders, such as ileus, bacterial overgrowth, functional dyspepsia and irritable bowel syndrome 1. SI contractions could be classified on the basis of their duration. Manometry is currently viewed as the best technique for diagnosing intestinal motor disorders. SI manometry gives a picture of the contractile activity of the gut over time by providing continuous recordings of intra-luminal pressures resulting from phasic contractions in the small bowel [2]. With the very recent invent of capsule endoscopy (CE) 3[4] physicians were allowed to record continuous information of SI activity, and to visualize and trace the whole variety of contraction patterns in a unique way. 
In this work, our purpose is to explore efficient methods for the automatic detection of frames registered during contractile activity. Visual annotation of contractions is an elaborating task, since the recording device of the capsule stores about 50,000 images and contractions might occur in undetermined time periods throughout the navigation course of the capsule in the intestinal tract.

Computer vision offers means to encode in quantitative patterns, 'scenes'that posse their own properties to be outstanding. Several techniques for the analysis of endoluminal images have been reported in the literature [567789].

However, addressing the automatic recognition of special patterns of contractions is a novel and challenging field in computer vision applications, and up to our knowledge it is not addressed in the bibliography. In this paper, we introduce an efficient feature extraction methodology based on anisotropic image processing for tracing frames of contractions, mining local directional information from the image gradient tensor. Feature evaluation in detecting positive examples of contractions has been performed by means of support vector machine classifier (SVM) [10.

The paper is organized as follows: Section 2 focuses on the method of feature extraction to characterize contraction patterns, section 3 discusses our results and the paper finishes with conclusions and future lines of research.

\section{Methodology for Feature Extraction and Classification of Contraction Patterns}

Contractions used to appear with a prolonged, strong occlusion of the lumen as shown in Fig 1, The omnipresent characteristic in these frames are the strong edges (wrinkles) of the folded intestinal wall, distributed in a radial way around the closed intestinal lumen.

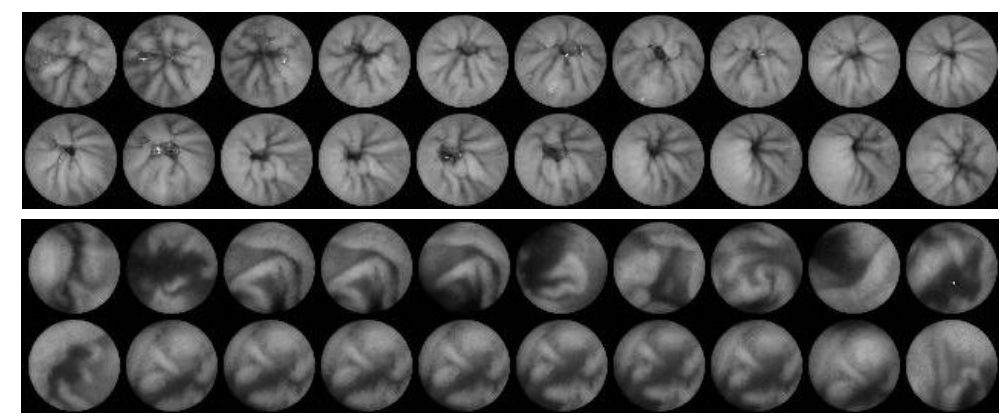

Fig. 1. Endoluminal images of positive (top) and negative examples (bottom)

The procedure to encode in a quantitative way the wrinkle-star pattern was accomplished in three steps. Firstly, the skeleton of the wrinkle pattern is extracted. Secondly, the center of the intestinal lumen is detected, as the point where the wrinkle edges converge using the image structure tensor. Finally, a set 
of descriptors were estimated taking into account the near radial organization of the wrinkle skeleton around the center of the intestinal lumen.

\subsection{Extraction of the Wrinkle Skeleton and Detection of the Center of the Intestinal Lumen}

To extract the wrinkle skeleton we used the local directional information of the image gradient tensor also called in the bibliography structural matrix (image structural tensor). The structural tensor of an image $\mathrm{J}$ is calculated as follows:

$$
J=\left[\begin{array}{ll}
J_{x x} & J_{x y} \\
J_{y x} & J_{y y}
\end{array}\right]
$$

where, subscripts indicate image derivatives [1].

Before estimating the structural matrix the image is smoothed by a $\sigma$-standard deviation $2 D$ Gaussian filter. The value of $\sigma$ was set equal to three, which was a good compromise between removing noisy edges and retaining local information in the scale of the wrinkle edges. The eigenvectors and the eigenvalues of the structural matrix provide rich information for the detection of the wrinkle edges. More specifically, the eigenvector that corresponds to the highest eigenvalue, is crossing the wrinkle edge, pointing large gradient variations. The second eigenvector is lying to the edge direction pointing towards the smallest gradient variations. Since the pattern of the contraction is a pattern with strong valley directionality, we used the gray matrix of the prime eigenvalue of the structure matrix for the initial detection of the wrinkle skeleton. An example is illustrated in Fig.2,

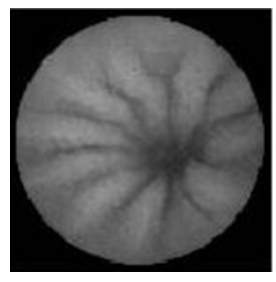

(a)

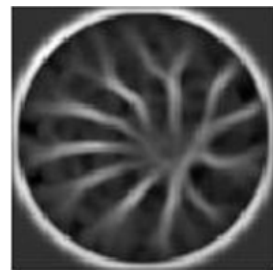

(b)

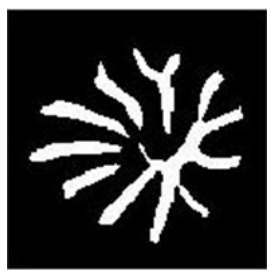

(c)

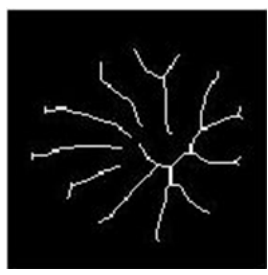

(d)

Fig. 2. (a) Original image, (b) gray-matrix of the first eigenvalues of the structural tensor, (c) thresholded binary image and (d) wrinkle skeleton

Since the center of the intestinal lumen serves as the center of symmetry for the wrinkle skeleton, the automatic detection of this point is a critical step in the whole procedure of the wrinkle-pattern extraction. In other words, this point defines the small pool in which strong streams (wrinkle edges) converge. To define this point we smoothed the image with a $2 D$ Gaussian filter of $\sigma$ equal to 13. The purpose of using such a large $\sigma$ is due to the fact that we want to retain information related only with large scale structures in the image, such as the blob of the intestinal lumen. In this case, the eigenvalues of the structural tensor would take high values in the area of the predominant structure of the 
intestinal lumen. Using the point with the highest second eigenvalue is a good approximation for detecting the center of the intestinal lumen. An illustrative example is given in Fig, 3 .

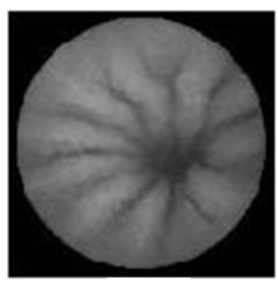

(a)

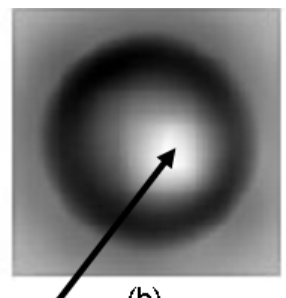

(b)

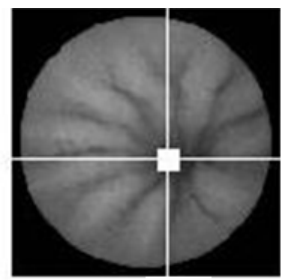

(c)

Point with highes

second eigenvalue

Fig. 3. (a) original image,(b) gray-matrix of the second eigenvalues of the structural tensor pointing the highest eigenvalue and (c) detection of the wrinkle pool

\subsection{Descriptors of Wrinkle Skeletons}

Our visual perception of a strong wrinkled-contraction is a star-wise wrinkle skeleton, co-central to the intestinal lumen. In other words, we have the presence of strong edges directed towards a central point, and uniformly spread around this point. To capture this feature of the star-pattern, we extracted a set of eight descriptors as follows: First considering the center of intestinal lumen as the center of wrinkle symmetry, we divide the wrinkle skeleton in four quadrants (see Fig. 4). In each quadrant we construct the histogram of the orientation of the second eigenvector estimated in the skeleton pixels. Since we are interested in tolerating small differences and keeping the general tendency of skeleton orientation, our histogram used just four bins corresponding to the directions $0^{\circ}, 90^{\circ}, 135^{\circ}, 45^{\circ}$. Consequently, we have a pattern of four descriptors in each quadrant. As it is illustrated in the example of Fig. 4, in the case of near symmetric star-wise pattern, the directional information in quadrant 1 is matching the directional information in quadrant 3. Equally, the directional information in quadrant 2 is similar with the directional information in quadrant 4 . Therefore, for the whole, wrinkle skeleton, we result in 8 descriptors by summing the directional information of quadrants 1 and 3 , and the direction information of quadrants 2 and 4, resulting in a set of 8 descriptors. Figure 4, illustrates the construction of the set of descriptors for a synthetic star-wise pattern. The histogram directional information in quadrant $1,2,3$ and 4 is: $\{132,132,132,0\}$, $\{132,132,0,132\},\{132,132,132,0\}$ and $\{132,132,0,132\}$ respectively. Thus, the resulted pattern for the whole skeleton is: $\{264,264,264,0,264,264,0,264\}$.

In addition to the set of the eight descriptors giving information about the orientation of wrinkles, we are interested in defining the entropy of the skeleton features. In particular, four more descriptors -one for each quadrant- were extracted measuring the local directional information of wrinkle edges. Since the 


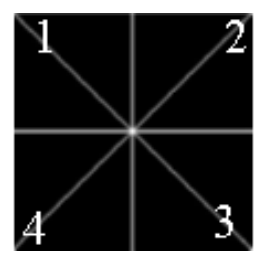

Fig. 4. Skeleton of an ideal star-wise pattern

second eigenvector gradient fields are following the direction of image valleys, local directional information on wrinkle edges can be described by estimating the tendency of the direction of second eigenvector fields for the skeleton points. More specifically, for each quadrant, the entropy of the direction of second eigenvectors is estimated for the skeleton points. In a pure wrinkled contraction, in each quadrant of the wrinkle skeleton, the entropy should be low. For a regular frame where no contractile activity of the lumen is evident, the local directional information in the extracted skeleton is less ordered, resulting in high entropy values. An example is given in Fig 5 and Fig 6 , Figure 5 , shows a wrinkle pattern and the entropy obtained is: $\{0.34,0.37,0.24,0.33\}$. Figure 6, shows two negative examples and the obtained entropy is: $\{0.46,0.36,0.58,0.48\}$ and $\{0.47,0.32,0.59,0.55\}$, for Fig 6(a) and Fig 6(b) respectively. Finally, we include two parameters to measure the sharpness of the wrinkle edges. From the structural matrix J (1), we define two more descriptors, a and b estimated from the skeleton points. These descriptors are given as follows [12]:

$$
\begin{gathered}
a=\operatorname{trace}\{J\} \\
b=\left(a^{2}-4|J|\right) * \sqrt{\left(\left|a^{2}-4\right| J||\right)}
\end{gathered}
$$

Descriptors a and b were calculated at each skeleton point and averaged over all points. Well defined crisp edges with predominant directionality tend to have high values for a and b parameters, whereas skeletons resulted from smoothed gradient variations have lower values. An example is given in Fig[7

Finally, the resulted pattern for the wrinkle skeleton comprised of 14 features: a set of 8 directional features, a set of 4 local entropy related features and 2 features of edge sharpness.

\section{Results}

The proposed feature extraction methodology was evaluated, using a set of images from 9 videos obtained using endoluminal capsules developed by Given Imaging Ltd, Israel [13. The data were provided from the Digestive Diseases Department of the General Hospital de Vall d' Hebron, Barcelona Spain. Our data contained 521 positive examples of images of contractions and 619 images not corresponding to intestine contractions (called negative examples). The negative 


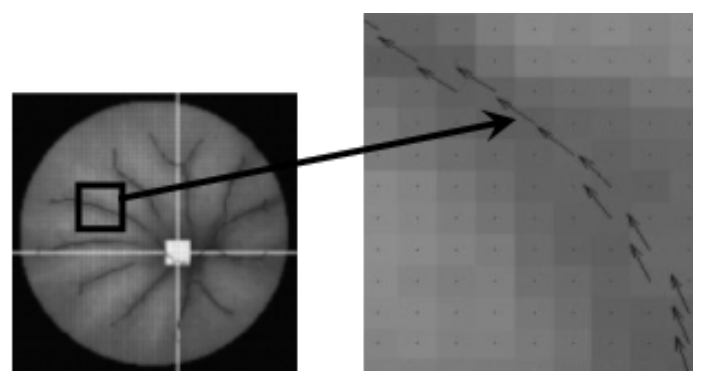

Fig. 5. A wrinkle pattern and the second eigenvectors gradient fields following the wrinkle edges. The well ordered vectors result in low entropy values in each quadrant.

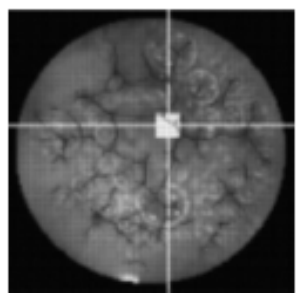

(a)

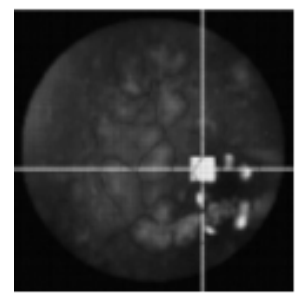

(b)

Fig. 6. Negative examples, with rich edge information organized in a random way

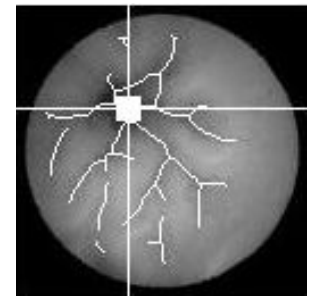

(a)

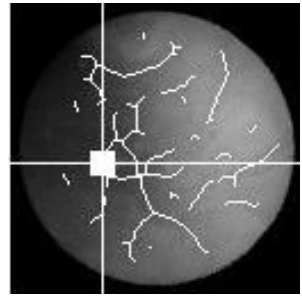

(b)

Fig. 7. (a) Well defined crisp wrinkles from a contraction frame $\{a, b\}=\{0.71,0.82\}$, (b) regular frame with smoothed wrinkle edges $\{a, b\}=\{0.21,0.05\}$

examples were selected in order to represent different phenomena occurring in the intestine (turbid presence, empty intestine without contraction, images with lumen appearance (partially or totally visible), images without lumen appearance, etc). Classification performance was tested by means of a SVM classifier with radial basis function kernel and employing the hold out cross validation method. The hold out cross validation method was simulated for 100 times, using for training each time $50 \%$ of the data from each data set and testing with the remaining. The classification performance was estimated in terms of sensitivity 
and specificity. On average the system detected correctly $90.84 \%$ of the positive examples, and $94.43 \%$ of the negative examples (Table 11). For a comparative evaluation, Table 2 reports the performance of the $k$-nearest neighbor (KNN) classifier on the same task.

Table 1. Classification accuracies estimated using the SVM classifier

\begin{tabular}{ccc}
\hline & Sensitivity(\%) & Specificity(\%) \\
\hline Mean Value & 90.84 & 94.43 \\
Standard Deviation & 1.8 & 1.29 \\
\hline
\end{tabular}

Table 2. Classification accuracies estimated using the KNN classifier

\begin{tabular}{ccc}
\hline & Sensitivity(\%) & Specificity(\%) \\
\hline Mean Value & 92.38 & 89.48 \\
Standard Deviation & 1.50 & 2.58 \\
\hline
\end{tabular}

\section{Discussion}

In this paper we presented a feature extraction methodology for the detection of specific configurations characterizing the wrinkled contractions in video capsule endoscopy. We proposed an approach based on image structural matrix, to extract informative skeletons, to obtain the center of the intestinal lumen and to estimate sets of informative descriptors. We extracted three sets of descriptors that our intuition can easy interpret. First, we showed a simple model for extracting directional information in four directions, which model adequately, pure, star-symmetric patterns. However, endoluminal images have rich texture and frequently, skeletons are contaminated from edge information that do not correspond to wrinkle contractions. A second set of local directional information was used to add information of homogeneous and well directed valleys. Contractions are not always pure symmetric patterns, and the model of symmetric information in quadrants defined from the center of contraction seemed to give incomplete information. For this, we have added in our pattern two more descriptors, related with the sharpness of the extracted wrinkle edges.

Concluding, anisotropic image processing combined with statistic measurements and classification techniques offer elegant means for modelling and characterizing motility information in endoluminal images. Our results in testing sets of endoluminal images suggest the feasibility of the method as an assisting tool for the detection of contractions.

\section{Acknowledgments}

This work was supported in part by a research grant from Given Imaging Ltd., Yoqneam Israel, H. U. Vall d'Hebron, Barcelona, Spain, as well as the 
projects FIS-G03/1085, FIS-PI031488, TIC2003-00654 and MI-1509/2005. The technology and methods embraced by this disclosure has been filed for patent protection.

\section{References}

1. Kellow, J.E., Delvaux, M., Aspriroz, F., Camilleri, M., Quigley, E.M.M., Thompson, D.G.: Principles of applied neurogastroenterology: physiology/motilitysensation. Gut 45(2) (1999) 1117-1124

2. Hansen, M.B.: Small intestinal manometry. Physiological Research 51 (2002) $541-556$

3. Idden, G., Meran, G., Glukhovsky, A., Swain, P.: Wireless capsule endoscopy. Nature (2000) 541-556

4. Rey, J.F., Gay, G., Kruse, A., R, L.: European society of gastrointestinal endoscopy guideline for video capsule endoscopy. Endoscopy 36 (2004) 656-658

5. Tjoa, M.P., Krishnan, S.M.: Feature extraction for the analysis of colon status from the endoscopic images. Biomedical Engineering OnLine 2 (2003) 3-17

6. Karkanis, S.A., Iakovidis, D.K., Maroulis, D.E., Karras, D.A., Tzivras, M.: Computer aided tumor detection in endoscopic video using color wavelet features. IEEE Trans-actions on Information Technology in Biomedicine 7 (2003) 141-152

7. Magoulas, G., Plagianakos, V., Vrahatis, M.: Neural network-based colonoscopic diagno-sis using online learning and differential evolution. Applied Soft Computing 4 (2004) 369-379

8. Kodogiannis, V.S., Chowdrey, H.S.: Multi-network classification scheme for computer-aided diagnosis in clinical endoscopy. In: Proceedings of the International Conference on Medical Signal Processing (MEDISP), Malta (2004) 262-267

9. Boulougoura, M., Wadge, V., Kodogiannis, V.S., Chowdrey, H.S.: Intelligent systems for computer-assisted clinical endoscopic image analysis. In: Proceedings of the 2nd IASTED Conference on Biomedical Engineering, Innsbruck, Austria (2005) 405-408

10. Vapnik, V.N.: The Nature of Statistical Learning Theory. Springer (1995)

11. Cyganek, B.: Combined detector of locally-oriented structures and corners in images based. LNCS 2658 (2003) 721-730

12. Acar, B., Beaulieu, C.F., et al.: Edge displacement field-based classification for improved detection of polyps in ct colonography. IEEE Transactions on Medical Imaging 21(12) (2002) 1461-1467

13. Given Imaging, Ltd: (http://www.givenimaging.com/. 2005) 